\title{
Scale exhaustivity and the Modification Condition*
}

\author{
M. Ryan Bochnak \\ University of California, Berkeley
}

\begin{abstract}
This paper pursues a Vector Space Semantics (VSS) analysis of evaluative and extreme adjectives in absolute and comparative constructions, with a particular emphasis on the licensing of measure phrases (MPs) in these environments. I show that the Modification Condition (Winter 2005), which restricts the distribution of MPs with locative/directional PPs and dimensional adjectives, can be extended to account for MP licensing with evaluative and extreme adjectives as well. Importantly, the non-satisfaction of the Modification Condition is entailed when a set of vectors does not exhaust the range of possible values on a particular scale. This observation thus allows us to link a long-standing generalization that scale exhaustivity and MP licensing are crucially related (Bierwisch 1989) with the formal denotational properties of certain linguistic expressions.
\end{abstract}

Keywords: measure phrases, gradable adjectives, comparatives, vector space semantics, scale exhaustivity, cross-categorial modification

\section{The distribution of measure phrases}

It is well known in the literature on degree semantics that there are co-occurrence restrictions between measure phrases (MPs) and dimensional adjectives (DAs). As shown in (1), positive-polar DAs can co-occur with MPs, while negative-polar DAs cannot (Bierwisch 1989; Kennedy 2001; Sassoon 2010). These polarity-based restrictions are only observed with the absolute (unmarked) form of the adjectives. With their corresponding comparative forms, this distinction is neutralized, as shown in (2). Furthermore, when MPs are licensed, in both the absolute and comparative forms, there is no norm-related inference, as observed in (3).

10 meters tall/*short/wide/*narrow/deep/*shallow

(2) 10 meters taller/shorter than the John Hancock Tower

* I am grateful to Helena Aparicio, Andrea Beltrama, Eva Csipak, Chris Kennedy, and Asia Pietraszko for comments on this work. I would also like to thank the audiences at SALT 23, especially Manfred Krifka, Louise McNally, Jessica Rett, and Galit Sassoon, as well as three anonymous SALT reviewers for thoughtful comments and questions. All remaining errors are my own. This work was supported in part by NSF grant \#1155196.

(C)2013 Bochnak 
Scale exhaustivity

(3) a. Fred is 3 feet tall. $\nrightarrow$ Fred is tall.

b. Fred is 5 inches shorter than Dale. $\nrightarrow$ Fred is short.

A somewhat less familiar observation is that MPs also display co-occurrence restrictions with locative and directional prepositional phrases (PPs), as shown in (4) (Zwarts 1997; Zwarts \& Winter 2000).

10 meters beside/outside/*near/*on the house

Based in part on the MP facts, researchers in the Vector Space Semantics framework (VSS: Zwarts 1997; Zwarts \& Winter 2000) have sought a unified analysis of locative/directional PPs on one hand, and DAs on the other hand. In this framework, vectors (directed line segments) are taken to be semantic primitives whose endpoints locate an individual's location in space or on a scale. Under this view, both DAs and PPs denote sets of vectors (Faller 2000; Winter 2005; Schwarzschild 2012). These can combine intersectively with MPs, which also denote sets of vectors (more formal details on VSS are given below). The Modification Condition in (5) is posited to account for the range and limits of MP licensing with PPs and DAs:

(5) The Modification Condition (MC): An expression that is associated with a set of vectors $W$ can be modified by an MP only if $W$ is non-empty, upward and downward monotone, and does not contain zero vectors. (Winter 2005)

In this paper, I aim to extend the account to evaluative adjectives (EvAs, e.g., pretty; Bierwisch 1989) and extreme adjectives (ExAs, e.g., gigantic; Paradis 2001; Morzycki 2012). These classes of adjectives have received somewhat less attention in the literature, and yet are also subject to co-occurrence restrictions with MPs. I show that by adopting the additional assumption that certain adjectives have contextually-valued zeros, the basic account using the $\mathrm{MC}$ in (5) can be extended in such a way that explains MP (non-)co-occurrence and related patterns found with EvAs and ExAs. Specifically, I show that these properties fall out from the fact that such adjectives denote sets of vectors that are not downward monotone, which is in turn entailed by the fact that they do not exhaust the range of values on a particular scale. That the VSS analysis can be extended to EvAs and ExAs so straightforwardly serves as an argument for VSS framework in general, as well as the analytical intuitions that are so easily imported into it in order to account for the data discussed in this paper. ${ }^{1}$

This paper proceeds as follows. In section 2 I outline the relevant data on MP licensing with EvAs and ExAs. I then present more background on the VSS framework in section 3, briefly outlining how it models the semantics of locative/directional PPs and DAs, as well as how the MC is used to account for MP

1 Thanks to an anonymous SALT reviewer for framing the issues in this way. 
licensing in those cases. I then extend the analysis to EvAs and ExAs in sections 4 and 5, respectively. I discuss the cross-linguistic landscape of MP licensing in section 6, and consider the options for reconciling MC with recent literature on MP licensing within degree-based frameworks in section 7. Section 8 concludes.

\section{Measure phrases with evaluative and extreme adjectives}

First considering EvAs like pretty, these do not occur with MPs in the absolute form. It is important to note that this restriction is not due to the fact that there are no conventionalized units for measuring non-dimensional scalar concepts. As shown in (6), nonce MPs are acceptable in comparatives.

(6) Context: In a beauty contest where contestants are given scores ranging from 0 to 10 stars, Contestant $\mathrm{A}$ is given a score of 7 stars and Contestant B is given a score of 5 stars.

a. * Contestant A is 7 stars pretty.

b. Contestant A is 2 stars prettier than Contestant B.

As for ExAs like gigantic, these also do not license MPs, even when their nonextreme counterparts do, as shown in (7). Furthermore, as shown in (8), comparatives with ExAs reject MPs as well. While comparatives with ExAs are somewhat degraded compared to their non-extreme counterparts (Cruse 1986), to the extent that they are acceptable, they carry a norm-related inference. Comparatives with non-extreme adjectives are not associated with such an inference (Bierwisch 1989; Rett 2007).

(7) Context: Fred has a height of 8 feet - he is downright gigantic!

a. Fred is 8 feet tall/*gigantic.

a. * Fred is 1 foot more gigantic than Dale.

b. ? Fred is more gigantic than Dale. $\rightarrow$ Dale is gigantic.

There is thus no general conceptual difficulty that prohibits measurement with non-dimensional adjectives. Rather, there seems to be a semantic restriction with EvAs and ExAs that prohibits MPs from occurring with the absolute form, and for ExAs with the comparative as well. Before proposing a solution to this puzzle, I introduce in the next section the main machinery of VSS and how it accounts for the data in (1)-(4). 
Scale exhaustivity

\section{Vector Space Semantics and the Modification Condition}

The VSS framework was originally conceived to model the interpretation of locative and directional PPs (Zwarts 1997; Zwarts \& Winter 2000). ${ }^{2}$ The main insight of this framework is the addition of vectors (directed line segments) to the domain. Some of the formal properties of vectors that we will make use of in this paper are outlined in (9), adapted from Zwarts \& Winter (2000), where $V$ is a set of vectors $v$.

(9) Let $\langle V, 0,+, \cdot\rangle$ be a vector space over the real numbers $\mathbb{R}$
a. $\langle a, b\rangle$ is a located vector $v \in V$ with start point $a$ and end point $b$
b. $\forall s \in \mathbb{R}$ and $\forall v \in V:(s \cdot v) \in V$ (scalar multiplication)
c. $|v|:$ the norm of $v$; associates $v \in V$ to a non-negative $s \in \mathbb{R}$ (its length)

\subsection{Prepositional phrases}

In this system, PPs denote sets of vectors that point in a certain direction relative to some reference object (the object of the preposition). Informally, the denotation of the PP outside the house can be modeled as in (10). ${ }^{3}$

(10) $\llbracket$ outside the house $\rrbracket=\lambda v \cdot v$ points outwards from the boundary of the house

The sentence The chair is outside the house is then true if there exists a vector $v$ in the set denoted by (10) such that (the eigenspace of) some contextually salient and unique chair is located at the endpoint of $v$.

MPs also denote sets of vectors, as shown in (11). Specifically, they denote sets of vectors that have a particular length, which is named by the MP. MP+PP constructions are then interpreted intersectively, as in (12).

$$
\begin{aligned}
& \llbracket 10 \text { meters } \rrbracket=\lambda v \cdot|v|=10 \mathrm{~m} \\
& \llbracket 10 \text { meters outside the house } \rrbracket=\lambda v \cdot \llbracket 10 \text { meters } \rrbracket(v) \wedge \llbracket \text { outside the house } \rrbracket(v)
\end{aligned}
$$

Licit $\mathrm{MP}+\mathrm{PP}$ constructions are subject to the Modification Condition (MC) in (5), repeated below as (13). The statement of MC relies on a particular notion of monotonicity defined in (14). In essence, the set denoted by a PP must be closed under lengthening or shortening. A set of vectors is upward monotone if a

2 For reasons of space, I only outline the basic formal properties of vectors here. Readers are referred to the papers cited for a more rigorous introduction to the system.

3 Throughout this paper, I write denotations in terms of characteristic functions rather than sets, even though in the main text I will usually describe certain constituents as denoting sets. I have greatly simplified the semantics of PPs in order to avoid introducing many of the formal details used by Zwarts \& Winter (2000), which would take us too far afield. 
lengthening of a vector in the set is also a member of that set. Likewise, a set of vectors is downward monotone if a shortening of a vector in the set is also a member of that set.

(13) The Modification Condition (MC): An expression that is associated with a set of vectors $W$ can be modified by an MP only if $W$ is non-empty, upward and downward monotone, and does not contain zero vectors.

(14) Upward (downward) monotone: A set of vectors $A \subseteq V$ is upward (downward) monotone iff for all vectors $v \in A$ and $w \in V$, if $v \leq w(v \geq w)$ then $w \in A$. (Winter 2005)

MC correctly predicts that (12) is licit, since the set denoted by outside the house is both upward and downward monotone. Any lengthening or shortening of a vector in the set also results in a vector in that set. That is, supposing a chair is located outside the house, moving it closer to or farther away from the house does not make it false that it is outside the house. Meanwhile, MC blocks illicit MP+PP combinations such as *10 meters near the house. PPs headed by near impose a length restriction on the vectors that are members of the sets they denote. Supposing that a chair counts as near the house, moving it farther away from the house may make it false that it is still near the house. That is, the vectors in the set near the house are not upward monotone, thereby ruling out MPs in this case.

\subsection{Dimensional adjectives and comparatives}

DAs in VSS also denote sets of vectors oriented along the dimension that is lexically associated with the adjective (Faller 2000; Winter 2005). For each dimension $D$, we define a unit vector $u_{D}$ such that $\left|u_{D}\right|=1$. Let $t$ be a variable over scalar multipliers. The set of vectors denoted by the underlying form of the DA tall can be modeled as in (15), where $H$ represents the dimension of height.

$$
\llbracket t a l l \rrbracket=\lambda v \cdot v=\left\langle 0, t \cdot u_{H}\right\rangle: t>0
$$

Under this analysis, a slight modification of Faller's, the underlying form of tall simply denotes the set of upward-pointing vectors along the height dimension. As is standardly argued in much work on the semantics of degree constructions (Cresswell 1976; von Stechow 1984; Heim 1985; Kennedy 1997, among many others), both the unmarked absolute form and the comparative-marked form of DAs are derived from a more basic underlying source, namely the kind in (15). Following Kennedy (1997), among others, assume that the contribution of a null absolute morpheme is to introduce a relation to a standard relative to which an absolute (i.e., unmarked) construction is interpreted. Let $t_{0}$ be the standard value for the DA, and the semantics 
for the absolute form of tall, rendered as tall $a b s$, is given in (16), where $t_{0}$ is the standard. It is this form of the DA that MPs combine with (Kennedy 1997). In bare absolute constructions (e.g., Fred is tall), the standard value is supplied by context.

$$
\llbracket \text { tall }_{a b s} \rrbracket=\lambda v \cdot v=\left\langle 0, t \cdot u_{H}\right\rangle: t>t_{0}>0
$$

Since it is this form of the DA that MPs combine with, it is at this stage where MC applies. The set in (16) is upward monotone: a lengthening of a vector in the set is also a vector in that set. The set in (16) is also downward monotone, provided that $t_{0}=0$. Thus, the conditions for MC are met, and MPs are licensed with tall, so long as the standard value for tall is set to 0 . This fact has an important consequence. In setting the standard to 0 , we get the welcome result that there is no norm-related interpretation for the MP construction (or rather, we obtain trivial norm-relatedness, since any object with a non-zero height counts as tall).

In the case of negative-polar adjectives, the underlying form of the adjective denotes the set of downward-pointing vectors along the dimension of height, as in (17). Here, downward-ness is represented by multiplying the unit vector by the scalar -1. Once again, the derived absolute form also denotes a set of vectors in relation to a standard, as in (18).

$$
\begin{aligned}
& \llbracket \text { short } \rrbracket=\lambda v \cdot v=\left\langle\infty, t \cdot-u_{H}\right\rangle: t>0 \\
& \llbracket \text { short }_{a b s} \rrbracket=\lambda v \cdot v=\left\langle\infty, t \cdot-u_{H}\right\rangle: t>t_{0}>0
\end{aligned}
$$

Note that the conditions for $\mathrm{MC}$ are not met for the set of vectors denoted by short $_{a b s}$. The set is upward monotone: any lengthening of a vector in the set yields a vector in that set. ${ }^{4}$ However, the set is not downward monotone for any finite $t_{0}$. MC therefore cannot apply, correctly ruling out MP co-occurrence with negative polar adjectives.

We now turn to comparatives with dimensional adjectives. Recall from (2) that the distinction between positive and negative polar adjectives is neutralized in this environment, and MPs are allowed with both types of DAs in the comparative. Following Faller (2000) and Winter (2005), comparative phrases denote sets of difference vectors. That is, they denote sets of vectors on a derived scale on the relevant dimension, where the zero and standard values are set to the endpoint of a vector determined by the than phrase. For both taller and shorter, this would be the height of the standard of comparison. In (19), the comparative morpheme takes a set of vectors $W$ (the set denoted by the underlying form of the gradable adjective), and a standard $s$ introduced by the than phrase, and returns a set of difference vectors.

4 The set is upward monotone if we allow vector endpoints to be below 0 (i.e., we allow for the possibility that objects may have a negative height). We can avoid making this counterintuitive claim by stipulating that dimensional scales only range over $\mathbb{R}^{+}$. See Faller 2000 for discussion. 
The set of vectors denoted by the phrase taller than Dale is given in (20), where $h_{d}$ represents Dale's maximal height (the endpoint of the height vector associated with Dale). ${ }^{5}$

$$
\begin{aligned}
& \llbracket \text {-er } \rrbracket=\lambda W \lambda s \lambda v \cdot W(v) \wedge v=\left\langle e p t(s), t \cdot u_{\operatorname{dim}(W)}\right\rangle: t \in(0, \infty) \\
& \begin{aligned}
\llbracket \text { taller than Dale } \rrbracket & =\lambda v \cdot v=\left\langle 0, t \cdot u_{H}\right\rangle: t>0 \wedge v=\left\langle h_{d}, t \cdot u_{H}\right\rangle: t \in(0, \infty) \\
& =\lambda v \cdot v=\left\langle h_{d}, t \cdot u_{H}\right\rangle: t \in(0, \infty) \quad \text { (by set intersection) }
\end{aligned}
\end{aligned}
$$

The set of vectors in (20) is both upward and downward monotone. Any lengthening or shortening of a vector in the set is also a vector in that set. The conditions for MC are therefore met, correctly predicting compatibility with MPs.

Comparatives with the negative-polar DAs can also occur with MPs. The denotation of shorter than Dale is given in (21). This set of vectors is also upward and downward monotone, so the conditions for MC are met, and MPs are predicted to be licit.

$$
\llbracket \text { shorter than Dale }=\lambda v \cdot v=\left\langle h_{d}, t \cdot-u_{H}\right\rangle: t \in(0, \infty)
$$

This theory of measure phrase licensing couched within the VSS framework thus accounts for a range of facts related to the distribution of MPs with prepositional phrases and dimensional adjectives in their absolute and comparative forms. In order to give this theory even more bite, we should attempt to extend the framework to other classes of adjectives. In the following sections, I build off the analysis for absolute and comparative DAs to extend the theory to evaluative and extreme adjectives to account for the facts in (6)-(8).

\section{Evaluative adjectives}

Recall that evaluative adjectives do not combine with MPs in the absolute form, but do combine with MPs in the comparative; see (6). The reason is that the set of vectors denoted by the absolute form of EvAs is upward monotone for any standard, but not downward monotone for any standard. However, the set denoted by the comparative form is both upward and downward monotone. Thus, by MC, MPs are blocked with the absolute form, but acceptable in the comparative.

To formalize this idea, I follow Sassoon (2011) in drawing a distinction between absolute and relative zero values for a scale. A scale's zero value is absolute if that value constitutes the zero value for that scale in any context, ${ }^{6}$ and that value marks

5 To simplify the discussion, I gloss over how the standard vector is derived from the than phrase. See Faller 2000 and Winter 2005 for the formal details.

6 Sassoon states this condition in terms of possible worlds as indices on the interpretation of gradable predicates. 
the complete absence of a property. For example, the zero value for tall is absolute, namely 0 height: it marks the complete absence of height. Otherwise, a zero value is relative, or context-dependent.

Sassoon's intuition is that EvAs have relative zeros, rather than absolute ones. In particular, it is nonsensical to ask what amount of beauty one must have to count as minimally pretty, namely a 0 amount of beauty. The zero value for EvAs like pretty does not coincide with the property of lacking any measure of beauty. Rather, it is the point at which entities begin to have a noticeable amount of a property, a minimally significant amount. Just as the standard for relative adjectives like tall can vary from context to context, so too can the relative zero values of EvAs. Some evidence that the contextual zeros of such scales are distinct from their contextual standard values comes from the modifier somewhat. For scales where the zero and standard values coincide, e.g., for minimum-standard adjectives like open, a sentence containing somewhat entails the same sentence without the modifier. With EvAs, the entailment does not go through. This contrast is illustrated in (22).

(22) a. The door is somewhat open. $\rightarrow$ The door is open.

b. Contestant B is somewhat pretty. $\nrightarrow$ Contestant B is pretty.

The contrast between scales with absolute and relative zeros is schematized in (23), where $0_{A}$ and $0_{C}$ are absolute and context-dependent zeros, respectively. ${ }^{7}$

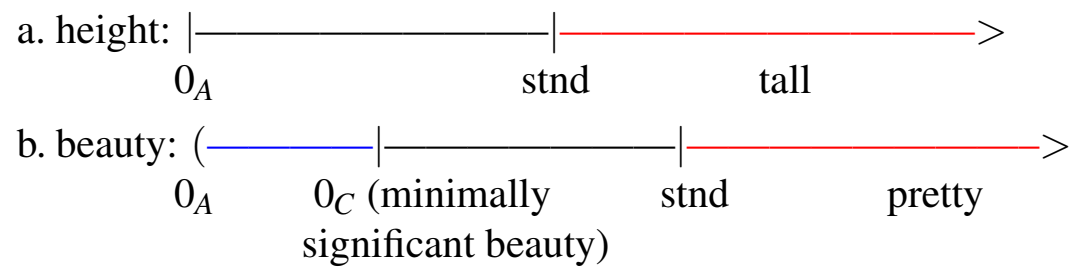

With this picture of scale structure for EvAs in mind, we can model the denotation of the absolute form of pretty as in (24). It denotes the set of upward-pointing vectors on the scale of beauty that start at absolute zero, but whose standards must be above a contextual, relative zero.

$$
\llbracket \text { pretty }_{a b s} \rrbracket=\lambda v \cdot v=\left\langle 0_{A}, t \cdot u_{B}\right\rangle: t>t_{0}>0_{C}
$$

7 In principle, a scale with a relative zero could have an absolute zero, or could lack one. For instance, fat seems to behave like an EvA, and so would have a contextual zero, even though there is an absolute zero on the scale, namely 0 weight (or perhaps more generally 0 size). For the scale of beauty, it is unclear whether we want to say that there is an absolute zero, or whether it simply has no lower bound. I set aside this issue for now, and use a scale for beauty that has an absolute zero, but one that is not included in the scale. 
This set of vectors is upward monotone for any standard, but not downward monotone for any standard. Even if we fix the standard to the contextual zero value, the set is not downward monotone. MP co-occurrence is therefore ruled out by MC.

Note that the fact that EvAs like pretty do not in their absolute form denote sets of vectors that are downward monotone follows from a more general observation that has been made about such adjectives, namely that they do not range over all possible values of the scale. That is, EvAs are not downward-monotone because they do not exhaust all possible values of their scales. That there is a connection between scale exhaustivity and MP co-occurrence has been noted in the literature (Bierwisch 1989; Sassoon 2011; Breakstone 2012), but has not received any formal treatment. Under the present account, non-exhaustivity automatically entails that the sets of vectors denoted by such an adjective will be non-monotonic in the sense used here. Thus, in the case of EvAs, the observation that MP co-occurrence is related to scale exhaustivity finds an explanation in the MC. This connection is even more general, as will be shown in the discussion of ExAs in the next section.

The comparative form of EvAs can be modeled as in (25), where $b_{b}$ represents Contestant B's maximal amount of beauty (the endpoint of the beauty vector associated with Contestant B).

$$
\llbracket p r e t t i e r \text { than Contestant } B \rrbracket=\lambda v \cdot v=\left\langle b_{b}, t \cdot u_{B}\right\rangle: t \in(0, \infty)
$$

As was the case for the comparative forms for positive and negative DAs, the set of vectors denoted by the comparative phrase in (25) is both upward and downward monotone, and is thus compatible with MPs as predicted by MC.

Before concluding this section, two more comments are in order. First, note that negative DAs like short can actually be reduced to the class of EvAs. The height scale is upwardly unbounded, meaning that short lacks an absolute zero value. In fact, Sassoon (2011) analyzes negative DAs as having contextual zero values, namely the point at which we begin to notice a deficit in height. This connection seems not to be accidental. As pointed out by Rett (2007), negative-polar DAs are associated with a norm-related inference in certain degree constructions, such as how questions. This is a trait that they share with EvAs, but not positive-polar DAs, as shown in (26).

(26) a. How tall is Fred? $\nrightarrow$ Fred is tall.

b. How short is Dale? $\rightarrow$ Dale is short.

c. How pretty is Contestant A? $\rightarrow$ Contestant A is pretty.

It can therefore be taken as a welcome result that EvAs and negative DAs are modeled in the same way in the VSS account presented here.

Second, the data analyzed here require a revision of the Standard/Zero-Value Convention in Winter 2005. Winter recognizes that both the standard and zero 
values of an adjective may be contextually determined, but stipulates as part of the convention that only one of these at a time can be contextually determined. The convention is stated in (27), where $d_{S}$ is the standard value and $z_{S}$ is the zero value on a scale $S$.

(27) Standard/Zero-Value Convention: For a given scale $S, d_{S}=c_{S}$ iff $z_{S}=0$, and $z_{S}=c_{S}$ iff $d_{S}=0$, where $c_{S}$ is some [contextually-determined; MRB] vector in the closure of $S$. (Winter 2005)

Winter invokes this convention in his analysis of early and late. ${ }^{8}$ However, given the data from EvAs, the stipulation that only the zero value or the standard value may be contextually derived is too strict. In the case of EvAs, we must allow both the zero and standard values to be determined by context. Once we allow a contextual zero for EvAs, their behavior with respect to MPs in the absolute and comparative forms can be explained using the existing theoretical apparatus of VSS, and specifically the MC.

\section{Extreme adjectives}

Recall that extreme adjectives do not license MPs in the absolute or comparative forms, even when a non-extreme counterpart does license MPs. Furthermore, to the extent that they are acceptable, comparatives with ExAs always carry a norm-related inference. The relevant facts are repeated in (28)-(29).

(28) Context: Fred has a height of 8 feet - he is downright gigantic!

a. Fred is 8 feet tall/*gigantic.

a. * Fred is 1 foot more gigantic than Dale.

b. ? Fred is more gigantic than Dale. $\rightarrow$ Dale is gigantic.

The crucial observation here is that ExAs do not exhaust all possible values on the relevant scale (Paradis 2001; Morzycki 2012). In particular, they only range over values that are quite high on the associated scale. Under the particular implementation by Morzycki (2012), ExAs are restricted to points higher than some 'extreme' contextual standard, beyond which distinctions between such points are not salient. The basic intuition is schematized in (30), where the adjectives tall and gigantic share a scale, but gigantic has a higher standard value and ranges only over 'extreme' values on the scale. ${ }^{9}$

8 I do not treat these adjectives in this paper; see Winter 2005 for an analysis in the VSS framework. 9 Gigantic is not lexically restricted to the scale of height only. It can also range over extreme values of other dimensional scales, such as weight. 


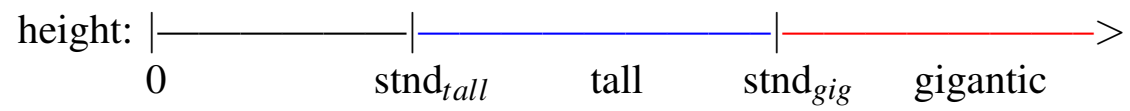

The connection to EvAs and negative-polar DAs should be clear. Those adjectives were shown to be non-monotonic in the sense intended here, thus ruling out MP co-occurrence in the absolute forms per MC. Non-monotonicity is in turn entailed by the fact that these adjectives do not exhaust all possible values on the relevant scale. To model the semantics of the absolute form of ExAs, I follow Morzycki's intuition that they are restricted to values above which particular points on the scale are no longer salient. The absolute form of gigantic is modeled as in (31), where $t_{c}$ is a variable over contextually salient scalar multipliers, and the function sup returns the supremum (least upper bound) of a set.

$$
\llbracket \text { gigantic }_{a b s} \rrbracket=\lambda v \cdot v=\left\langle 0, t \cdot u_{H}\right\rangle: t>\sup \left(\left\{t_{c}\right\}\right)
$$

The set of vectors denoted by (31) is upward monotone for any standard, but is not downward monotone for any standard, provided we make certain assumptions about possible values of $t_{c}$. In particular, we must assume that $\sup \left(\left\{t_{c}\right\}\right)$ cannot be 0 , which would make the set of vectors downward monotone. But allowing $\sup \left(\left\{t_{c}\right\}\right)$ to equal 0 would have two undesirable consequences. First, it would mean that all the values on the relevant scale are not salient, and therefore any degree of, say, height would count as extreme. Second, and relatedly, it would neutralize the difference in meaning between ExAs and their non-extreme counterparts. Recall that for non-extreme DAs like tall, the standard must be set to 0 to to license MPs, and allowing $\sup \left(\left\{t_{c}\right\}\right)$ for ExAs to equal 0 essentially reduces the semantics of ExAs to that of their non-extreme counterparts. Following Morzycki (2012), I assume that some notion of contextual domain restriction (e.g., von Fintel 1994) for salient values of the scale is necessary for the interpretation of ExAs.

Like the absolute form of EvAs and negative-polar DAs, the sets of vectors denoted by ExAs are non-monotonic, ruling out MP co-occurrence per MC. Furthermore, the more general notion of scale exhaustivity plays a crucial role once again: ExAs do not exhaust all possible values on the relevant scale. However, the similarities between EvAs and negative-polar DAs on one hand and ExAs on the other hand end here. Whereas the former group of adjectives allow MPs in comparatives, ExAs do not. Additionally, the comparative form of ExAs also gives rise to a norm-related inference, which is not the case for EvAs or negative-polar DAs. Comparative phrases with ExAs thus cannot be modeled following the template for EvAs and DAs.

I follow Morzycki in claiming that comparatives with ExAs carry a restriction on the values being compared, namely that the extremeness requirement of the absolute form carries over to the comparative. Within the degree-based framework 
assumed by Morzycki, this is modeled as a presupposition that the two degrees being compared both exceed the contextual salient points on the scale, i.e., both degrees are 'extreme'. In the VSS framework used here, we restrict the possible values for the endpoint of the standard vector. As a first pass, we can model a comparative phrase as in (32), where we simply add the extra restriction that the endpoint of the standard vector be above the contextually salient points on the scale. ${ }^{10}$

$$
\llbracket \text { more gigantic than Dale }=\lambda v \cdot v=\left\langle h_{d}, t \cdot u_{H}\right\rangle: t \in(0, \infty) \wedge h_{d}>\sup \left(\left\{t_{c}\right\}\right)
$$

(to be revised)

The semantics in (32) gets us norm-relatedness: Dale must count as gigantic even in the comparative. However, the set of difference vectors is both upward and downward monotone, just like comparatives with EvAs and DAs. Thus, MC does not preclude MPs from co-occurring with comparatives formed with ExAs. One possible solution to this issue would be to claim that it is not MC itself, but rather a lexical idiosyncrasy of gigantic that precludes MP co-occurrence with comparative ExAs. Indeed, as discussed in the following section, there are certain cross-linguistic idiosyncrasies in the distribution of MPs that cannot be accounted for by MC alone, and perhaps this is such a case as well. But such a solution seems unappealing, since, as we have previously observed, there seems to be a non-accidental connection between norm-relatedness and MPs not being licensed.

A second possible solution relates to an observation about comparatives with ExAs: they seem to be unacceptable in crisp judgment contexts. ${ }^{11}$ Comparatives with non-extreme DAs are acceptable in crisp judgment contexts, i.e., where there is only a slight difference between two objects in the degree to which they hold the relevant property (Kennedy 2007). ExAs do not seem to be acceptable in such cases, as shown in (33).

Context: Dale's height $=8$, and Fred's height $=8$ ' 0.5"

a. Fred is taller than Dale.

b. ?? Fred is more gigantic than Dale.

The restriction that the standard count as gigantic is satisfied, but the comparative is still odd if Fred is only slightly taller than Dale. This means that the difference set denoted by the comparative phrase crucially does not contain all possible vectors that begin at Dale's height. That is, the set does not exhaustively range over all possible values of the derived scale, and is correspondingly not downward monotone, ruling

10 I write this restriction as an extra conjunct in the truth conditions of (32), even though it might be best viewed as a presupposition, as in Morzycki 2012.

11 Thanks to Galit Sassoon for bringing this to my attention. 
out MP co-occurrence via MC. We can now update the semantics in (32) as in (34), where $\operatorname{diff}_{c}$ is a contextual difference value that a scalar multiplier must exceed.

$$
\llbracket \text { more gigantic than Dale } \rrbracket=\lambda v \cdot v=\left\langle h_{d}, t \cdot u_{H}\right\rangle: t>\operatorname{diff}_{c} \wedge h_{d}>\sup \left(\left\{t_{c}\right\}\right)
$$

This solution captures the fact that comparatives with ExAs are unacceptable in crisp judgment contexts. We then have a principled reason why MPs are not licensed: the relevant vector sets are not downward monotone, in violation of MC. However, this solution still does not directly capture the fact that the non-licensing of MPs seems to be linked to norm-relatedness. See Sassoon 2011 for further discussion of this link and possible avenues for future research.

\section{Lexical and cross-linguistic idiosyncrasies}

One may argue that a possible problem with relating the licensing conditions for MPs to MC is that it does not rule out certain MP+Adj combinations in English. For instance, it seems intuitive that the DA heavy should be modeled just like tall, only with a different specification for the dimension (weight instead of height).

$$
\llbracket h e a v y_{a b s} \rrbracket=\lambda v \cdot v=\left\langle 0, t \cdot u_{W}\right\rangle: t>t_{0}
$$

The set in (35) is both upward monotone for any standard, and downward monotone if the standard is 0 . But then it appears that MC incorrectly predicts that $* 20$ pounds heavy should be licit, and similarly for the DAs expensive and fast, which also do not license MPs in their absolute forms.

$\mathrm{I}$ argue that $\mathrm{MC}$ is only a necessary condition for MP licensing, but not a sufficient one, and that this is in fact a good thing, given certain cross-linguistic facts and idiosyncrasies. That there is much cross-linguistic variation in the licensing of MPs is well-known (e.g., Schwarzschild 2005). First, we observe that certain languages do allow MPs with the absolute form of those DAs where MC predicts they should be licit. The following examples from Norwegian and German come from Grano \& Kennedy (2012):

a. ei 200 kroners dyr lampe

$$
\text { a } 200 \text { kroner (*expensive) lamp }
$$

(Norwegian)

b. 100 Tonnen schwer

100 tons (*heavy)

(German)

c. 60 Stundenkilometer schnell

60 kilometers per hour (*fast)

(German)

Second, in many languages MPs are licensed with fewer DAs than English, and in the limiting case are banned altogether with the absolute form. Such languages 
include Japanese, Russian, and Spanish (see Schwarzschild 2005, and references therein). ${ }^{12}$

Despite the variation observed, there are nevertheless robust cross-linguistic generalizations regarding the distribution of MPs. For instance, MPs are generally allowed in the comparative forms of both positive and negative-polar DAs, even in languages that never allow MPs with the absolute form. Japanese is such a language; the data in (37) also come from Grano \& Kennedy (2012).

a. *2-meetoru segatakai

2-meter spine.high

Intended: '2 meters tall'

b. 2-meetoru sore yori segatakai

2-meters that than spine.high

' 2 meters taller than that'

Additionally, there seem to be no languages that allow MPs to co-occur with negative-polar DAs in the absolute form. EvAs and ExAs have generally not received as much attention in the literature, and further research needs to be carried out on these types of predicates and the possibilities for MP co-occurrence crosslinguistically. Grano \& Kennedy (2012) report that piaoliang 'pretty' in Mandarin can occur with a nonce MP in a so-called 'transitive comparative' construction, as shown in (38), where a 'milli-helen' is a unit of beauty needed to launch one ship. They do not report on whether this is also possible with the absolute form. The prediction made here is that it is not, but more research is needed to collect the relevant facts.

\section{Zhangsan piaoliang Lisi yi milli-helen.}

Zhangsan pretty Lisi one milli-helen

'Zhangsan is one milli-helen prettier than Lisi.' (Grano \& Kennedy 2012)

Thus, while MC is not a sufficient condition for ruling out certain MP+Adj combinations in particular languages, it appears to be a necessary condition for allowing such combinations both within and across languages. That is, MC crucially predicts where MP+Adj collocations should be ruled out on semantic grounds, and the predictions it makes in this respect seem to be cross-linguistically robust. However, languages still show some idiosyncrasy with respect to whether or not they license MPs with all the predicates that satisfy MC. Given the cross-linguistic variation, we still need to allow for some lexical stipulations on MP licensing with certain adjectives, e.g., to rule out $* 20$ pounds heavy in English.

12 See Sawada \& Grano 2011 for more details on the case of Japanese. 
Bochnak

\section{Implementing the Modification Condition}

We now come to the question of how MC should be implemented to account for the distribution of MPs. For Winter (2005), the application of MC is governed by a triviality filter on MP+Adj collocations, but as Winter himself points out, this essentially amounts to a restatement of MC. In this section I consider how MC might be implemented within two recent theories of MP distribution with DAs and comparatives, those of Schwarzschild (2005) and Svenonius \& Kennedy (2006), and also make comparisons between those proposals and the one argued for here. ${ }^{13}$

In Schwarzschild's (2005) system, MPs are predicates of gaps, rather than degree-denoting expressions as standardly assumed. This accounts for why MPs co-occur with comparatives so readily: comparison entails a gap between the relative positions on a scale of the objects being compared. That is, comparatives have a gap argument built into them that MPs have access to. Gradable adjectives in the absolute form, however, are the wrong type to combine with MPs: they have degree arguments, not gap arguments. Schwarzschild proposes an idiosyncratic type-shifting rule that applies only to those adjectives that combine with MPs. The type shift would for example apply to schwer 'heavy' in German, but not to heavy in English.

Under the VSS analysis proposed here, such a type-theoretic approach cannot be directly implemented. All gradable adjectives and comparative phrases denote (characteristic functions of) sets of vectors. MPs are also of this type, and are interpreted intersectively when licensed. For the sake of argument, let us consider how the MC might be implemented under Schwarzschild's approach. MC finds a natural place as a restriction on the domain of the semantic rule that allows adjectives in the absolute form to combine with MPs. Of course, there would still need to be some language-specific idiosyncrasy to account for why the rule can apply to schwer in German but not to heavy in English, but we still have a semantically-driven account for why adjectives like tall across languages can license MPs, but EvAs and ExAs can't. However, Schwarzschild's account predicts that MPs should be licensed with comparative ExAs, since these should have gap arguments just like any other comparative.

The account of Svenonius \& Kennedy (2006) also has a type-theoretical aspect, but at its core is essentially a syntactic account of MP licensing. Specifically, they propose that a phonologically null but syntactically present degree head is responsible for licensing MPs with comparative and absolute forms of gradable adjectives. On the type-theoretic side, they propose that gradable adjectives denote measure functions and consequently do not have a degree argument (Kennedy 1997).

13 The reader is referred to Grano \& Kennedy 2012 for a more detailed overview and evaluation of both of these theories, and the issues at stake in choosing between them. 
The role of degree morphemes is, in part, to introduce a degree argument that can be targeted by degree expressions like MPs. The degree head is therefore necessary on type-theoretic grounds to license MPs. On the syntactic side, the degree head has selectional restrictions that allow it to combine freely with comparatives, but only selectively with other gradable adjectives. The cross-linguistic idiosyncrasies in MP licensing are thus located in the selectional restrictions of this degree head across languages. Their evidence for the syntactic reality of this null degree head comes from the behavior of degree questions in Northern Norwegian. Grano \& Kennedy (2012) also argue for such a null syntactic head based on data from so-called transitive comparatives in Mandarin.

Once again, the type-theoretic aspects of this proposal are not relevant here. However, the syntactic nature of Svenonius \& Kennedy's analysis makes it easier to reconcile with the account proposed here. I have so far been agnostic as to what sort of syntax is at issue in MP constructions. But given the syntactic evidence for such a degree head across languages, we could propose a structure for the phrase five feet tall along the lines of (39).

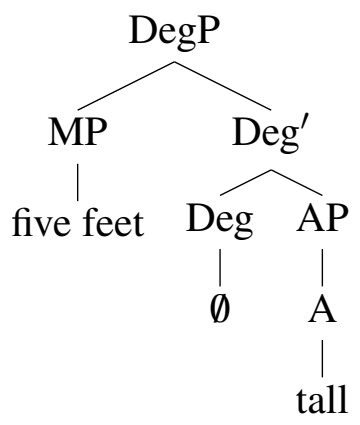

Unlike in Svenonius \& Kennedy's analysis, the degree head has no type-shifting consequences within the VSS account. Under the present account, the degree head should just denote the identity function. At the same time, Svenonius \& Kennedy do not directly address the question of whether the distribution of the degree head should be linked to the semantic properties of the predicates it selects. However, we have seen that $\mathrm{MC}$ is the relevant semantic property that restricts the distribution of MPs. Putting this all together, we can let the degree head encode the presupposition that MC holds of the set of vectors denoted by the adjective. The semantics of the degree head can thus be modeled as in (40), where I use the colon notation for modeling presuppositional content, following Heim \& Kratzer (1998).

$$
\llbracket \emptyset \rrbracket=\lambda W: \operatorname{MC}(W) \cdot \lambda v \cdot W(v)
$$

This style of analysis allows us to overcome three problems. First, we previously had no way of accounting for why certain adjectives that in principle should allow 
MPs nevertheless do not, and the related cross-linguistic facts. Whereas the adjective heavy satisfies MC, it is not within the domain of the selectional restrictions of the degree head in English. Across languages, the degree head can select for different sets of adjectives, and the cross-linguistic differences are simply due to different syntactic selectional restrictions of the degree head. Second, there was the problem faced by Svenonius \& Kennedy of how to relate the distribution of the degree head to the semantic properties of the predicates it selects. This is implemented here as a presupposition on the application of the degree head. Third, we can explain the absence of MPs with comparatives with ExAs under this account more straightforwardly than under Schwarzschild's account. MPs must also be licensed by the same degree head in comparatives as well, and since, as argued in section 5, comparatives with ExAs do not satisfy MC, the presupposition of the degree head is therefore not satisfied in this case.

At this point, one more issue presents itself, namely the cross-categorial distribution of MPs. Indeed, the fact that MPs occur with PPs, gradable adjectives, and comparatives was the main motivation for pursuing a unified semantic analysis in this paper and by previous authors working in VSS. Presumably, then, we would want to generalize the syntax in (39) to PPs as well. I leave this issue to future research, though see Morzycki 2006 for a starting point for generalizing the DegP syntax to PPs, and also to VPs with for X-time adverbials.

\section{Conclusion}

In this paper I have extended the general account for cross-categorial MP licensing to two more classes of adjectives-evaluative adjectives and extreme adjectives-in their absolute and comparative forms. I have shown that Winter's (2005) Modification Condition for the licensing of MPs can be straightforwardly applied to these cases by adopting insights from the recent literature on EvAs and ExAs. The key insight from the investigation is that scale exhaustivity is a driving force behind MP licensing. This idea that there is a connection between scale exhaustivity and MP licensing has been noted since at least Bierwisch (1989), and receives a principled account here in terms of the Modification Condition. That the analysis can be so easily extended to account for these cases provides an argument in favor of the development of a unified cross-categorial semantics (and syntax) for expressions that co-occur with MPs, and a unified analysis of the semantic restrictions on such combinations. Specifically, the VSS framework, which was originally motivated to account for the semantics of PPs, provides an intuitive means for analyzing the absolute and comparative forms of several classes of adjectives as well.

I have already highlighted two key points for future research. The first is more cross-linguistic research on MP licensing, especially with EvAs and ExAs. The 
Scale exhaustivity

second is whether a unified syntactic structure for degree constructions and PPs is also tenable, given the strong semantic parallels in these domains. Another important question that still needs to be explored is how to incorporate other types of modification into this system. For instance, the adverb very can occur with positive and negative-polar DAs and EvAs alike, so long as they encode a relative standard (Kennedy \& McNally 2005). ExAs, meanwhile, co-occur with a distinct class of modifiers such as downright and positively (Morzycki 2012). However, these modifiers are not interpreted intersectively, and so should receive a distinct analysis from the one proposed here for MPs. In any case, in order to show that an analysis in terms of vector spaces can be generalized to replace what has become the standard degree-based approach for gradable adjectives, further investigation into a wider range of degree constructions is essential. That such an analysis can support the range of facts outlined in this paper certainly points to the conclusion that this is a promising avenue to pursue in future research.

\section{References}

Bierwisch, Manfred. 1989. The semantics of gradation. In Manfred Bierwisch \& Ewald Lang (eds.), Dimensional Adjectives: Grammatical Structure and Conceptual Interpretation, 71-261. Berlin: Springer-Verlag.

Breakstone, Micha. 2012. Inherent evaluativity. In Ana Aguilar Guevara, Anna Chernilovskaya \& Rick Nouwen (eds.), Sinn und Bedeutung (SUB) 16, 113-126. Cambridge, MA: MIT Press.

Cresswell, Max J. 1976. The semantics of degree. In Barbara Partee (ed.), Montague Grammar, 261-292. New York: Academic Press.

Cruse, Allan. 1986. Lexical Semantics. Cambridge: Cambridge University Press.

Faller, Martina. 2000. Dimensional adjectives and measure phrases in vector space semantics. In Martina Faller, Stefan Kaufmann \& Marc Pauly (eds.), Formalizing the Dynamics of Information, 151-170. Stanford, CA: CSLI Publications.

von Fintel, Kai. 1994. Restrictions on quantifier domains. Amherst, MA: University of Massachusetts dissertation.

Grano, Thomas \& Christopher Kennedy. 2012. Mandarin transitive comparatives and the grammar of measurement. Journal of East Asian Linguistics 21. 219-266. doi:10.1007/s10831-012-9090-Y.

Heim, Irene. 1985. Notes on comparatives and related matters. Ms. University of Texas at Austin.

Heim, Irene \& Angelika Kratzer. 1998. Semantics in Generative Grammar. Blackwell.

Kennedy, Christopher. 1997. Projecting the adjective: The syntax and semantics of gradability and comparison: University of California, Santa Cruz dissertation. 
Kennedy, Christopher. 2001. Polar opposition and the ontology of 'degrees'. Linguistics and Philosophy 24. 33-70. doi:10.1023/A:1005668525906.

Kennedy, Christopher. 2007. Modes of comparison. In Malcolm Elliott, James Kirby, Osamu Sawada, Eleni Staraki \& Suwon Yoon (eds.), Chicago Linguistic Society (CLS) 43, Chicago: Chicago Linguistic Society.

Kennedy, Christopher \& Louise McNally. 2005. Scale structure, degree modification and the semantics of gradable predicates. Language 81(2). 345-381. doi:10.1353/lan.2005.0071.

Morzycki, Marcin. 2006. Atelicity and measure phrases: Licensing measure phrase modification across AP, PP, and VP. In Donald Baumer, David Montero \& Michael Scanlon (eds.), 25th West Coast Conference on Formal Linguistics (WCCFL), 279-287. Somerville, MA: Cascadilla Proceedings Project.

Morzycki, Marcin. 2012. Adjectival extremeness: Degree modification and contextually restricted scales. Natural Language and Linguistic Theory 30(2). 567-609. doi:10.1007/s11049-011-9162-0.

Paradis, Carita. 2001. Adjectives and boundedness. Cognitive Linguistics 12. 47-65. doi:10.1515/cog1.12.1.47.

Rett, Jessica. 2007. Antonymy and evaluativity. In Tova Friedman \& Masayuki Gibson (eds.), Semantics and Linguistic Theory (SALT) 17, 210-227. Ithaca, NY: CLC Publications.

Sassoon, Galit Weidman. 2010. The degree functions of negative adjectives. Natural Language Semantics 18. 141-181. doi:10.1007/s11050-009-9052-8.

Sassoon, Galit Weidman. 2011. Be positive! Norm-related implications and beyond. In Ingo Reich, Eva Horch \& Pauly Dennis (eds.), Sinn und Bedeutung (SUB) 15, 531-546. Saarbrücken, Germany: Saarland University Press.

Sawada, Osamu \& Thomas Grano. 2011. Scale structure, coercion, and the interpretation of measure phrases in Japanese. Natural Language Semantics 19. 191-226. doi:10.1007/s11050-011-9070-1.

Schwarzschild, Roger. 2005. Measure phrases as modifiers of adjectives. Recherches Linguistiques de Vincennes 34. 207-228.

Schwarzschild, Roger. 2012. Directed scale segments. In Anca Chereches (ed.), Semantics and Linguistic Theory (SALT) 22, 65-82. Ithaca, NY: CLC Publications.

von Stechow, Arnim. 1984. Comparing semantic theories of comparison. Journal of Semantics 3. 1-77. doi:10.1093/jos/3.1-2.1.

Svenonius, Peter \& Christopher Kennedy. 2006. Northern Norwegian degree questions and the syntax of measurement. In Mara Frascarelli (ed.), Phases of Interpretation, 133-161. The Hague: Mouton de Gruyter.

Winter, Yoad. 2005. Cross-categorial restrictions on measure phrase modification. Linguistics and Philosophy 28. 233-267. doi:10.1007/s10988-004-1469-4.

Zwarts, Joost. 1997. Vectors as relative positions: A compositional semantics of 
Scale exhaustivity

modified PPs. Journal of Semantics 14. 57-86. doi:10.1093/jos/14.1.57.

Zwarts, Joost \& Yoad Winter. 2000. Vector space semantics: A model-theoretic analysis of locative prepositions. Journal of Logic, Language, and Information 9. 169-211. doi:10.1023/A:1008384416604.

M. Ryan Bochnak

Department of Linguistics

1203 Dwinelle Hall

University of California, Berkeley

Berkeley, CA 94720-2650

USA

bochnak@berkeley.edu 